\title{
A Worst-case Evaluation Method for Dynamic Systems
}

\author{
Wen-Hou Ma Huei Peng \\ Department of Mechanical Engineering \\ and Applied Mechanics \\ University of Michigan \\ Ann Arbor, MI 48109-2125 \\ hpeng@umich.edu
}

\begin{abstract}
A worst-case evaluation method is presented in this paper. The objective of this method is to identify worst-case maneuvers so that the performance of dynamic systems under extreme conditions can be evaluated. Depending on the dynamics and information structure of the system, the worst-case evaluation problems can be classified into four sub-cases. Classical optimal control and game theories are used to construct algorithms to obtain linear solutions analytically. When the plant and/or the control algorithm is nonlinear, the true worst-case solution can be obtained from numerical methods. Two case-study examples are presented. A linear example presents the time domain and frequency domain results comparing the four linear algorithms. The generation of the worst-case steering and braking maneuver to rollover an articulated vehicle is then presented as a "real" application example. Interested reader can download the PC-based software and generate the simulation results by visiting the website http://arc.engin.umich.edu/sw_distri/arcsim.html.
\end{abstract}

\section{Introduction}

Automatic control theories have made tremendous progress in the past three decades. Control analysis and synthesis methodologies have been extended from linear, single-input-single-output (SISO), nominal stability consideration to nonlinear, multiinput-multi-output (MIMO) and robust performance. Control systems are now an integral part of many complex engineering systems, and have greatly improved their efficiency, safety and convenience. While tools exist for the design, analysis and simulation of these control systems, many mission-critical control systems have been evaluated/fine-tuned in a brute-force manner. This brute-force evaluation process is very time-consuming because one must ensure that these control systems work satisfactorily under all possible scenarios. This process usually involves extensive experiments and/or simulations, and may take up to dozens of work-years to accomplish. Further, there is no guarantee that the worst-case scenario has been identified. These two weaknesses demonstrate the need for a more systematic evaluation methodology. Such methodology should identify the weak link of the plant/control systems, and generate worst-case scenarios intelligently.

In this paper, we propose a worst-case evaluation criterion and present associated design methodologies. It should be emphasized that we are not proposing a control design method and claim that the controller should be optimized under the worst-case scenarios. Rather, the methodology proposed is a evaluation process to make sure that the performance of the dynamic system is satisfactory under worst-case scenarios. One example that clearly shows the importance of performance under worst-case scenarios is 
the 1992 Isuzu trooper episode. The Consumer Report rated the safety of Isuzu-Trooper "unacceptable" since it rolls over under a test maneuver. Isuzu countered that the maneuver is too extreme and rarely occurs in real world. Nevertheless, its sales plummeted more than $53 \%$ in one year.

There are two major theoretical contributions by this paper: (1) a systematic worst-case evaluation procedure is presented; and (2) a two-player game theory with preview information structure is solved. Two-player game theory has been widely used to study the dynamic interactions of two opposing intelligent bodies, especially in the areas such as economics, politics, and war games for several decades. In the modern control field, game theory has been used for the robust control design since the 1960's (e.g., Dorato and Drenick, 1964, Witsenhausen 1968, Yaesh and Shaked 1989). The design objective is to find the control algorithm that minimizes a given performance index under worst-case disturbances. When the performance index is of linear-quadratic type, the result was found to be equivalent to an $H_{\infty}$ control problem (Basar and Bernhard, 1995). In this paper, we apply the two-player game theory to the evaluation of vehicle control systems, in which case the vehicle control systems and the worst-case scenario generator are described as two intelligent bodies with opposing goals.

Preview information, which is often utilized in control designs, has not been fully explored in the development of two-player game theories. When preview information is available to one of the players, we have either the control advantaged case or the disturbance advantaged case. In the control advantaged case, the control law has access to the disturbance signal within a finite preview window (e.g. forward-looking vision systems can preview future road curvature). The control algorithm depends partially on the feedforward information, rather than relies on large feedback gains. When the control signal is previewable (e.g. due to actuator delays), we have the disturbance advantaged case. It is necessary to solve the preview game theories since actuator delays frequently exist in control systems.

For most dynamic systems, worst-case scenarios usually occur in the regions where nonlinearities cannot be neglected. Therefore, it will be necessary to extend the worst-case methodology to cover nonlinear systems. Existing nonlinear $H_{\infty}$ algorithms (e.g. Isidori and Astofi 1992, Lu and Doyle 1993, Van der Schaft 1992) are useful tools for generating worst-case disturbances for nonlinear systems. Numerical solutions, on the other hand, are much more important in practice. A major advantage of numerical solutions is that they can be applied to a wide varieties of nonlinear systems. Nonlinearities such as look-up tables, if-then rules, hysteresis and so forth are difficult to be described/solved through analytical techniques. These nonlinearities, however, are commonly included in modern simulation models. A finite horizon optimal solution, with proper initial guess for optimal disturbances (based on analytical solutions, engineering intuitions, etc.) could be used to solve worst-case disturbances for general nonlinear systems numerically.

The remainder of this paper is organized as follows: Section 2 contains the problem statement. In section 3, solutions of the four worst-case categories are 
presented. The numerical algorithms to compute nonlinear worst-case disturbances will be presented in section 4. Simulation results for two example systems are presented in section 5. Finally, conclusions are made in section 6 .

\section{Problem Statement}

The purpose of the worst-case evaluation methodology is to generate worst-case disturbances for dynamic systems which might include control sub-systems. In other words, the methodology should cover both 1-player (worst-case disturbance only) and 2player (disturbance and control) cases. In general, the nonlinear plant may have the form

$$
\underline{\dot{x}}=f(\underline{x}, \underline{u}, \underline{w}, t)
$$

where $\underline{x}$ is the state vector, $\underline{u}(t)$ is the control vector and $\underline{w}(t)$ is the disturbance vector. Instead of dealing with the worst-case disturbance generation problem for general nonlinear dynamic systems shown in Eq.(1), we selected an easier route. First, the problems are divided into four cases according to the plant dynamics and information structure. The linear time-invariant solutions of the four cases are then obtained. All the plant nonlinearities and/or time-varyingness are then taken care of numerically. Analytical solutions from the linear algorithms serve as "good initial guesses" which improve the chances of obtaining the global optimal solution numerically. The linearized time-invariant model of Eq.(1) is assumed to have the following state space representation:

$$
\underline{\dot{x}}(t)=A \underline{x}(t)+B \underline{u}(t)+D \underline{w}(t)
$$

These control $(\underline{u}(t))$ and disturbance $(\underline{w}(t))$ players have contradicting goals, in the sense that $\underline{u}(t)$ tries to minimize, while $\underline{w}(t)$ tries to maximize the cost function

$$
J\left(t_{o}, \underline{u}, \underline{w}\right)=\frac{1}{2} \int_{t_{0}}^{t_{f}}\left\{\underline{x}(t)^{T} Q \underline{x}(t)+\underline{u}(t)^{T} R \underline{u}(t)-\underline{w}(t)^{T} P \underline{w}(t)\right\} d t
$$

In the 1-player case, the control player is absent from the game, and the optimization problem is to find $\underline{w}(t)$ which maximizes the following cost function

$$
J\left(t_{0}, \underline{w}\right)=\frac{1}{2} \int_{t_{0}}^{t_{f}}\left\{\underline{x}^{T}(t) Q \underline{x}(t)-\underline{w}^{T}(t) P \underline{w}(t)\right\} d t
$$

The cost function might be modified when preview information exists, but is always assumed to have the quadratic form shown in Eqs.(3) and (4). Q, R, and P are weighting matrices that are positive semi-definite $(\mathrm{Q})$ and positive definite (R and P), respectively. This requirement is meant to make the cost function strictly convex-concave, so that the optimal solution is unique. In this paper, the derivation will be performed based on finite horizon cases. The algebraic solutions will be implemented to generate the initial guess in the simulations. This is, however, strictly due to their easier implementation, and should not be viewed as an implication that we are solving infinite-horizon problems. 


\section{Four cases of the worst-case problems}

Depending on the problem architecture and the information available to the control and/or disturbance player, the worst-case disturbance generation problems can be classified into the following four cases:

- One player without preview information (1P)

- One player with preview information (1PP)

- Two players without preview information (2P)

- Two players with preview information (2PP)

It should be pointed out that in the one-player cases, control signals may still present. However, they are not "in the game". In other words, when the control algorithm is not designed to optimize the cost function, one-player cases apply. The linear solutions of these four cases are summarized in the following.

\subsection{One-Player without Preview (1P)}

When we are evaluating a dynamic system either without control sub-systems, or with control sub-systems whose control laws (e.g. PID, LQ) are exactly known to the disturbance algorithm, it is appropriate to formulate a 1-player optimization problem. In the case control sub-systems do not exist, the optimization problem becomes:

Given

$$
\underline{\dot{x}}(t)=A \underline{x}(t)+D \underline{w}(t)
$$

Find the disturbance signal which maximizes the cost function

$$
J=\frac{1}{2} \int_{t_{0}}^{t_{f}}\left[\underline{x}(t)^{T} Q \underline{x}(t)-\underline{w}(t)^{T} P \underline{w}(t)\right] d t
$$

This problem is quite standard, and the optimal solution is known to be (Bryson and Ho 1975)

$$
\underline{w}(t)^{*}=-P^{-1} D^{T} K(t) \underline{x}(t)
$$

where $\mathrm{K}$ is solved from the Riccati-like equation

$$
\dot{K}(t)=-A^{T} K(t)-K(t) A+K(t) D P^{-1} D^{T} K(t)+Q
$$

The 1P problem formulation can also be applied to the evaluation of a dynamic system with control sub-systems. In this case, the disturbance signal is computed based on the augmented plant dynamics, and the augmented state matrix $A_{c}$ should be used instead of the openloop state matrix $A$ in Eqs.(5) and (8).

\subsection{One-Player with Preview (1PP)}

When the control law is unknown, but the control signal within a finite preview window is available to the disturbance player (e.g. due to actuator delay), we have a preview disturbance problem which is exactly dual to the classical LQ preview control problem (Tomizuka and Whitney 1975, Tomizuka 1975, Tomizuka 1976). For the sake 
of conciseness, we will only list the crucial equations below. The key trick of solving the preview problem is to assume that the optimal cost function can be described by :

$$
\begin{aligned}
J^{*}(t) & =\frac{1}{2} \underline{x}^{T}(t) K(t) \underline{x}(t)+\frac{1}{2} \int_{0}^{t_{l a}} \int_{0}^{t_{l a}} \underline{u}^{T}\left(t+l_{1}\right) K_{u}\left(t, l_{1}, l_{2}\right) \underline{u}\left(t+l_{2}\right) d l_{1} d l_{2} \\
& +\frac{1}{2} \underline{u}^{T}\left(t+t_{l a}\right) K_{d}(t) \underline{u}\left(t+t_{l a}\right)+\underline{x}^{T}(t) \int_{0}^{t_{l a}} F_{1}(t, l) \underline{u}(t+l) d l+\underline{x}^{T}(t) F_{2}(t) \underline{u}\left(t+t_{l a}\right)
\end{aligned}
$$

where $t_{l a}$ is the preview time, i.e., at time $t$, the control signal $u(\tau), \tau \in\left[t, t+t_{l a}\right]$ is known to the disturbance player. $K, K_{u}, K_{d}, F_{1}$ and $F_{2}$ are weighting matrices. This "generalized quadratic function" is assumed to be a good approximation of the true convex cost function in the neighborhood of the global optimal solution. Since we only know the control signal within the preview window, and intuitively the cost function depends on the control signal over the whole time horizon, certain assumption about future control signal is needed. A general dynamic form was commonly assumed (Tomizuka 1976):

$$
\underline{\dot{u}}(\tau)=A_{u} \underline{u}(\tau) \quad A_{u} \leq 0 \quad \tau \geq t+t_{l a}
$$

Frequently, $A_{u}$ is assumed to be zero, which means that the control signal is assumed to stay constant outside of the preview window. Under Eqs.(9) and (10), the optimal disturbance law can be obtained through standard Dynamic Programming techniques (Bryson and Ho 1975):

$$
\underline{w}^{*}(t)=-P^{-1} D^{T}\left[K(t) \underline{x}(t)+\int_{0}^{t_{l a}} F_{1}(t, l) \underline{u}(t+l) d l+F_{2}(t) \underline{u}\left(t+t_{l a}\right)\right]
$$

where the feedback and feedforward gains of this disturbance law are obtained from the following equations

$$
\begin{aligned}
& \dot{K}(t)=-A^{T} K(t)-K(t) A+K(t) D P^{-1} D K(t)+Q \\
& K\left(t_{f}\right)=0 \\
& \frac{\partial F_{1}(t, l)}{\partial t}=\frac{\partial F_{1}(t, l)}{\partial l}-\left(A^{T}-K(t) D P^{-1} D^{T}\right) F_{1}(t, l) \\
& F_{1}(t, 0)=K(t) B \\
& F_{2}(t)=-F_{1}\left(t, t_{l a}\right)-A^{T} F_{2}(t)+K(t) D P^{-1} D^{T} F_{2}(t)-F_{2}(t) A_{u} \\
& F_{2}\left(t_{f}\right)=0
\end{aligned}
$$

Detailed derivation of this optimization problem (albeit in a dual form) can be found from the literature (Tomizuka 1976, Peng and Tomizuka 1993) and thus are omitted here. It should be noted that Eq.(12) is exactly the same as Eq.(8). Also, Eq.(11) reduces to Eq.(7) when the preview time is zero.

\subsection{Two-Player without Preview (2P)}


In many applications, the control law may be either unknown or time-varying. Therefore, the one-player formulation described in sections 3.1-3.2 may not be applicable. The two-player game setting (Basar and Bernhard 1995) which prepares the disturbance player for its worst possible foe, seems a good alternative. The powerfulness of the two players can be specified by adjusting the weighting matrices ( $\mathrm{P}, \mathrm{Q}$ and $\mathrm{R})$ of the cost function shown in Eq.(3). The finite-time solution of this two-player game is well documented in the literature (Basar and Bernhard 1995) and the main results are summarized below. The optimal laws for the control and disturbance signals are

$$
\begin{aligned}
& \underline{w}^{*}(t)=P^{-1} D^{T} K(t) \underline{x}(t) \\
& \underline{u}^{*}(t)=-R^{-1} B^{T} K(t) \underline{x}(t)
\end{aligned}
$$

respectively, where the gain matrix $\mathrm{K}$ is solved from the following equation

$$
\begin{aligned}
& \dot{K}(t)=-A^{T} K(t)-K(t) A+K(t)\left(B R^{-1} B^{T}-D P^{-1} D^{T}\right) K(t)-Q \\
& K\left(t_{f}\right)=0
\end{aligned}
$$

\subsection{Two-Player with Preview (2PP)}

At first glance, preview and two-player assumptions seem to be contradictory to each other. When we have preview information, why couldn't we simply use the 1PP algorithm? When the preview time $t_{l a}$ is long enough, 1PP algorithm could be used. If the preview time is short, preview signal may not reveal the whole picture of the control player. Optimal disturbance law based on the 2PP algorithm takes the feedback algorithm of its opponent into consideration, and is more proper. Since the preview two player game theory is a new theoretical result, we will present both control-advantaged case and disturbance-advantaged case in the following.

When the disturbance signal is previewable to the control law, we have the control-advantaged case, the cost function to be optimized is modified to the following form

$$
J\left(t_{0}\right)=\frac{1}{2} \int_{t_{0}}^{t_{f}}\left[\underline{x}^{T}(t) Q \underline{x}(t)+\underline{u}^{T}(t) R \underline{u}(t)-\underline{w}^{T}\left(t+t_{l a}\right) P \underline{w}\left(t+t_{l a}\right)\right] d t
$$

With proper modification of the "generalized quadratic cost function", the optimal cost function for this case is assumed to be

$$
\begin{aligned}
J^{*}(t) & =\frac{1}{2} \underline{x}^{T}(t) K(t) \underline{x}(t)+\frac{1}{2} \int_{0}^{t_{l a}} \int_{0}^{t_{l a}} \underline{w}^{T}\left(t+l_{1}\right) K_{w}\left(t, l_{1}, l_{2}\right) \underline{w}\left(t+l_{2}\right) d l_{1} d l_{2} \\
& +\underline{x}^{T}(t) \int_{0}^{t_{l a}} F_{1}(t, l) \underline{w}(t+l) d l
\end{aligned}
$$

Define the Hamiltonian

$$
H \equiv \frac{1}{2}\left[\underline{x}^{T}(t) Q \underline{x}(t)+\underline{u}^{T}(t) R \underline{u}(t)-\underline{w}^{T}\left(t+t_{l a}\right) P \underline{w}\left(t+t_{l a}\right)\right]+\frac{d J^{*}(t)}{d t}
$$


The optimal algorithms can then be obtained based on the relationship $\left.\frac{\partial H}{\partial \underline{u}(t)}\right|_{\underline{u}^{*}(t)}=0$ and $\left.\frac{\partial H}{\partial \underline{w}\left(t+t_{l a}\right)}\right|_{\underline{w}^{*}\left(t+t_{l a}\right)}=0$. After tedious, but standard derivation based on the Dynamic Programming technique (Bryson and Ho 1975), the optimal disturbance and control laws were found to be

$$
\begin{aligned}
& \underline{w}^{*}\left(t+t_{l a}\right)=P^{-1} F_{1}\left(t, t_{l a}\right)^{T} \underline{x}(t) \\
& \underline{u}^{*}(t)=-R^{-1} B^{T}\left[K(t) \underline{x}(t)+\int_{0}^{t} F_{l a}(t, l) \underline{w}(t+l) d l\right]
\end{aligned}
$$

where

$$
\begin{aligned}
& \dot{K}(t)=-A^{T} K(t)-K(t) A+K(t) B R^{-1} B^{T} K(t)-F_{1}\left(t+t_{l a}\right) P^{-1} F_{1}\left(t+t_{l a}\right)^{T}-Q \\
& K\left(t_{f}\right)=0 \\
& \frac{\partial F(t, l)}{\partial}=\frac{\partial F(t, l)}{\partial}+\left(K(t) B R^{1} B^{T}-A^{T}\right) F(t, l)-F_{1}\left(t, t_{l a}\right) P^{-1} K_{w}\left(t, t_{l a}, l\right) \\
& F_{1}(t, 0)=K(t) D \\
& \frac{\partial K_{w}\left(t, l_{1}, l_{2}\right)}{\partial t}=\frac{\partial K_{w}\left(t, l_{1}, l_{2}\right)}{\partial l_{1}}+\frac{\partial K_{w}\left(t, l_{1}, l_{2}\right)}{\partial l_{2}}+F_{1}(t, l)^{T} B R^{-1} B^{T} F_{1}(t, l) \\
& K_{w}(t, 0, l)=D^{T} F_{1}(t, l)
\end{aligned}
$$

It should be noted that Eqs.(23) reduces to Eq.(17) when the preview time is zero. This can be shown simply by plugging in Eq.(24) to Eq.(23).

When the control signal is previewable to the disturbance law, we have the disturbance-advantaged case. The cost function to be optimized is then:

$$
J\left(t_{0}\right)=\frac{1}{2} \int_{t_{0}}^{t_{f}}\left[\underline{x}^{T}(t) Q \underline{x}(t)+\underline{u}^{T}\left(t+t_{l a}\right) R \underline{u}\left(t+t_{l a}\right)-\underline{w}^{T}(t) P \underline{w}(t)\right] d t
$$

For this case, the optimal cost function is assumed to have the form

$$
\begin{aligned}
J^{*}(t) & =\frac{1}{2} \underline{x}^{T}(t) K(t) \underline{x}(t)+\frac{1}{2} \int_{0}^{t_{l a}} \int_{0}^{t_{l a}} \underline{u}^{T}\left(t+l_{1}\right) K_{u}\left(t, l_{1}, l_{2}\right) \underline{u}\left(t+l_{2}\right) d l_{1} d l_{2} \\
& +\underline{x}^{T}(t) \int_{0}^{t_{l a}} F_{1}(t, l) \underline{u}(t+l) d l
\end{aligned}
$$

Follow a similar derivation process like the control advantaged case, the optimal laws were found to be

$$
\begin{aligned}
& \underline{w}^{*}(t)=P^{-1} D^{T}\left[K(t) \underline{x}(t)+\int_{0}^{t_{l a}} F_{1}(t, l) \underline{u}(t+l) d l\right] \\
& \underline{u}^{*}\left(t+t_{l a}\right)=-R^{-1} F_{1}\left(t, t_{l a}\right)^{T} \underline{x}(t)
\end{aligned}
$$

where 


$$
\begin{aligned}
& \dot{K}(t)=-A^{T} K(t)-K(t) A-K(t) D P^{-1} D^{T} K(t)+F_{1}\left(t+t_{l a}\right) R^{-1} F_{1}\left(t+t_{l a}\right)^{T}-Q \\
& K\left(t_{f}\right)=0 \\
& \frac{\partial F_{1}(t, l)}{\partial t}=\frac{\partial F_{1}(t, l)}{\partial l}-\left(K(t) D P^{-1} D^{T}+A^{T}\right) F_{1}(t, l)-F_{1}\left(t, t_{l a}\right) R^{-1} K_{u}\left(t, l, t_{l a}\right) \\
& F_{1}(t, 0)=K(t) B \\
& \frac{\partial K_{u}\left(t, l_{1}, l_{2}\right)}{\partial t}=\frac{\partial K_{u}\left(t, l_{1}, l_{2}\right)}{\partial l_{1}}+\frac{\partial K_{u}\left(t, l_{1}, l_{2}\right)}{\partial l_{2}}-F_{1}(t, l)^{T} D P^{-1} D^{T} F_{1}(t, l) \\
& K_{u}(t, 0, l)=B^{T} F_{1}(t, l)
\end{aligned}
$$

Again, it should be pointed out that Eq.(30) reduces to Eq.(17) when the preview time becomes zero.

\section{Nonlinear algorithms}

In many cases, nonlinear equations are necessary to describe the behavior of the dynamic systems accurately, based on which the worst-case maneuver can be computed. This is important since worst-case maneuvers usually occur in nonlinear regions. The nonlinear dynamic equations for the 1-player cases are assumed to be presented in the state-space form

$$
\underline{\dot{x}}=f(\underline{x}, \underline{w}, t) \quad \underline{x}(0)=\underline{x}_{O}
$$

where the initial time is assumed to be zero, and $\underline{x}_{o}$ is the initial condition of the state vector. The goal of the disturbance player is to maximize the performance index shown in Eq.(4). This is again a standard optimization problem, and the solution is known to be (Bryson and Ho 1975):

$$
\underline{w}(t)=-P^{-1} \underline{\lambda}(t) \frac{\partial f(t)}{\partial \underline{w}(t)}
$$

where the dynamics of the costate vector $\underline{\lambda}$ is governed by

$$
\underline{\dot{\lambda}}(t)^{T}=\underline{x}(t)^{T} Q-\underline{\lambda}(t)^{T} \frac{\partial f(t)}{\partial \underline{x}(t)} \quad \underline{\lambda}\left(t_{f}\right)=0
$$

For most nonlinear problems, analytical solution of this optimization problem is difficult to obtain. A two-point-boundary-value-problem (TPBVP) thus needs to be solved. Many modern simulation programs frequently include complex interaction among submodels. In other words, the nonlinear function $f(\underline{x}, \underline{w}, t)$ may not be presented in concise equation forms. The partial derivatives needed to for the TPBVP $\left(\frac{\partial f(t)}{\partial \underline{w}(t)}\right.$ and $\left.\frac{\partial f(t)}{\partial \underline{x}(t)}\right)$ thus need to be obtained numerically. The numerical procedure to solve the TPBVP is summarized in the following: 
- Step 1, start with initial guess of the optimal disturbance (e.g. from 1P or 1PP linear solutions). Solve Eq.(33) forward in time to obtain the states within the time horizon $\left[0, t_{f}\right]$.

- Step 2, compute $\frac{\partial f(t)}{\partial \underline{w}(t)}$ and $\frac{\partial f(t)}{\partial \underline{x}(t)}$ from the approximation

$$
\begin{aligned}
& \frac{\partial f(t)}{\partial \underline{w}(t)} \approx \frac{f(t, \underline{x}, \underline{w}+\Delta \underline{w})-f(t, \underline{x}, \underline{w}-\Delta \underline{w})}{2 \Delta \underline{w}} \\
& \frac{\partial f(t)}{\partial \underline{x}(t)} \approx \frac{f(t, \underline{x}+\Delta \underline{x}, \underline{w})-f(t, \underline{x}-\Delta \underline{x}, \underline{w})}{2 \Delta \underline{x}}
\end{aligned}
$$

by perturbing the vehicle dynamics artificially. The perturbation should be small enough so that Eqs.(36) and (37) remain good approximations, but large enough so that the search direction takes system nonlinearities into consideration.

- Step 3, Solve Eq.(35) backwards in time to obtain $\underline{\lambda}(t)$ from time $t_{f}$ to time 0 .

- Step 4, calculate the new disturbance $\underline{w}(t)$ from Eq.(34).

- Step 5, Check the convergence of $\underline{w}(t)$. Go back to Step 2 if necessary.

For two-player cases, the optimal disturbance algorithm is still generated by Eqs.(34) and (35), except that the nonlinear dynamics is now affected by both control and disturbance inputs (i.e., $\dot{x}=f(\underline{x}, \underline{u}, \underline{w}, t))$. The associated optimal algorithm for the control player is $\underline{u}(t)=R^{-1} \lambda(t) \frac{\partial f(t)}{\partial \underline{u}(t)}$. The partial derivative $\frac{\partial f(t)}{\partial \underline{u}(t)}$ can be obtained numerically in the same manner as described in Eqs.(36).

\section{Simulation Results}

Two example worst-case evaluation problems are presented in this section. In sub-section 5.1, results of a linear automatic steering problem is given. Results of this example highlight the difference among the four linear analytical solutions. A nonlinear, example is given in sub-section 5.2. This nonlinear example involves the generation of worst-case disturbance signals to rollover an articulated truck.

\subsection{Automatic steering example}

The target control system to be evaluated in this section is an automatic steering control system (Peng and Tomizuka 1990). The following fourth order model (commonly known as the bicycle model in the literature) is assumed to describe the vehicle lateral dynamics:

$$
\frac{d}{d t}\left[\begin{array}{c}
y_{r} \\
\dot{y}_{r} \\
\psi-\psi_{d} \\
\dot{\psi}-\dot{\psi}_{d}
\end{array}\right]=\left[\begin{array}{cccc}
0 & 1 & 0 & 0 \\
0 & \frac{A_{1}}{V} & -A_{1} & \frac{A_{2}}{V} \\
0 & 0 & 0 & 1 \\
0 & \frac{A_{3}}{V} & -A_{3} & \frac{A_{4}}{V}
\end{array}\right]\left[\begin{array}{c}
y_{r} \\
\dot{y}_{r} \\
\psi-\psi_{d} \\
\dot{\psi}-\dot{\psi}_{d}
\end{array}\right]+\left[\begin{array}{c}
0 \\
B_{1} \\
0 \\
B_{2}
\end{array}\right] u(t)+\left[\begin{array}{c}
0 \\
A_{2}-V^{2} \\
0 \\
A_{4}
\end{array}\right] w(t)
$$

where $y_{r}$ is the lateral deviation of the mass center of the vehicle from the reference (e.g. lane center), $\psi$ is the yaw angle of the vehicle, $\psi_{\mathrm{d}}$ is the desired yaw angle defined by the road. $u$ (the control) is the vehicle front wheel steering angle, and $w$ (the disturbance) is 
the road curvature. The elements of the state and input matrices are functions of vehicle parameters, and are known to be

$$
\begin{aligned}
& A_{1}=\frac{2\left(C_{\alpha f}+C_{\alpha r}\right)}{m} A_{2}=\frac{2\left(C_{\alpha r} a-C_{\alpha f} b\right)}{m} A_{3}=\frac{2\left(C_{\alpha r} a-C_{\alpha f} b\right)}{I_{z}} \\
& A_{4}=\frac{2\left(C_{\alpha f} a^{2}+C_{\alpha r} b^{2}\right)}{I_{z}} B_{1}=\frac{-2 C_{\alpha f}}{m} B_{2}=\frac{-2 a C_{\alpha f}}{I_{z}}
\end{aligned}
$$

where $C_{\alpha f}$ and $C_{\alpha r}$ are the cornering stiffness of the front and rear tires, respectively. $a$ and $b$ are the distance between vehicle c.g. to the front and rear axles, respectively. $m$ is the vehicle mass, and $I_{z}$ is the vehicle yaw moment of inertia. The objective of the disturbance player (which controls $w$ ) is to maximize the output signal

$$
y=y_{r}+d_{S}\left(\psi-\psi_{d}\right)=\left[\begin{array}{llll}
1 & 0 & d_{S} & 0
\end{array}\right] \underline{x}
$$

which corresponds to the lateral deviation of a point at a distance, $\mathrm{d}_{\mathrm{s}}$, ahead of the vehicle mass center. The nominal values of the vehicle parameters are listed in Table 1.

Table 1 Nominal values of the vehicle parameters

\begin{tabular}{|l|c|c|c|c|c|c|}
\hline Parameter & $\mathrm{m}(\mathrm{kg})$ & $\mathrm{Iz}(\mathrm{kg}-\mathrm{m} 2)$ & $C_{\alpha}(\mathrm{N} / \mathrm{rad})$ & $\mathrm{V}(\mathrm{m} / \mathrm{sec})$ & $\mathrm{a}, \mathrm{b}(\mathrm{m})$ & $\mathrm{ds}(\mathrm{m})$ \\
\hline Nominal Value & 1573 & 2782 & $\begin{array}{c}46000(\mathrm{f}) \\
38850(\mathrm{r})\end{array}$ & 32 & $1.034,1.491$ & 1.9 \\
& & & & & \\
\hline
\end{tabular}

The simulation scenario is as following: the vehicle starts from a non-zero initial tracking error $\left(\mathrm{y}=0.5 \mathrm{~m}, \psi-\psi_{d}=0\right)$. The control algorithm for the front wheel steering angle is LQ-based (i.e., $u(t)=-K_{c} \underline{x}$ ). The road curvature $\mathrm{w}(\mathrm{t})$ is computed from the four algorithms presented in section 3. In addition, it is limited by a saturation function (minimum radius of curvature $=200$ meter). In the state penalty matrix $Q$, only $y$ is penalized.

Figure 1 shows the tracking error and disturbance signals of the 1P algorithms (based on both openloop state matrix $A$ and closed-loop state matrix $A_{c}=A-B K_{c}$ ). The openloop (i.e. $\mathrm{w}=0$ ) case is also presented for comparison. It can be seen that when the disturbance law is based on openloop state matrix $A$, it is too weak compared with the control player, and simply gives up (not a good worst-case algorithm). When the disturbance is designed based on $A_{c}$, it does something quite intuitive: maximum curvature will be used; and fast switching between the two extreme values "at the right time". 

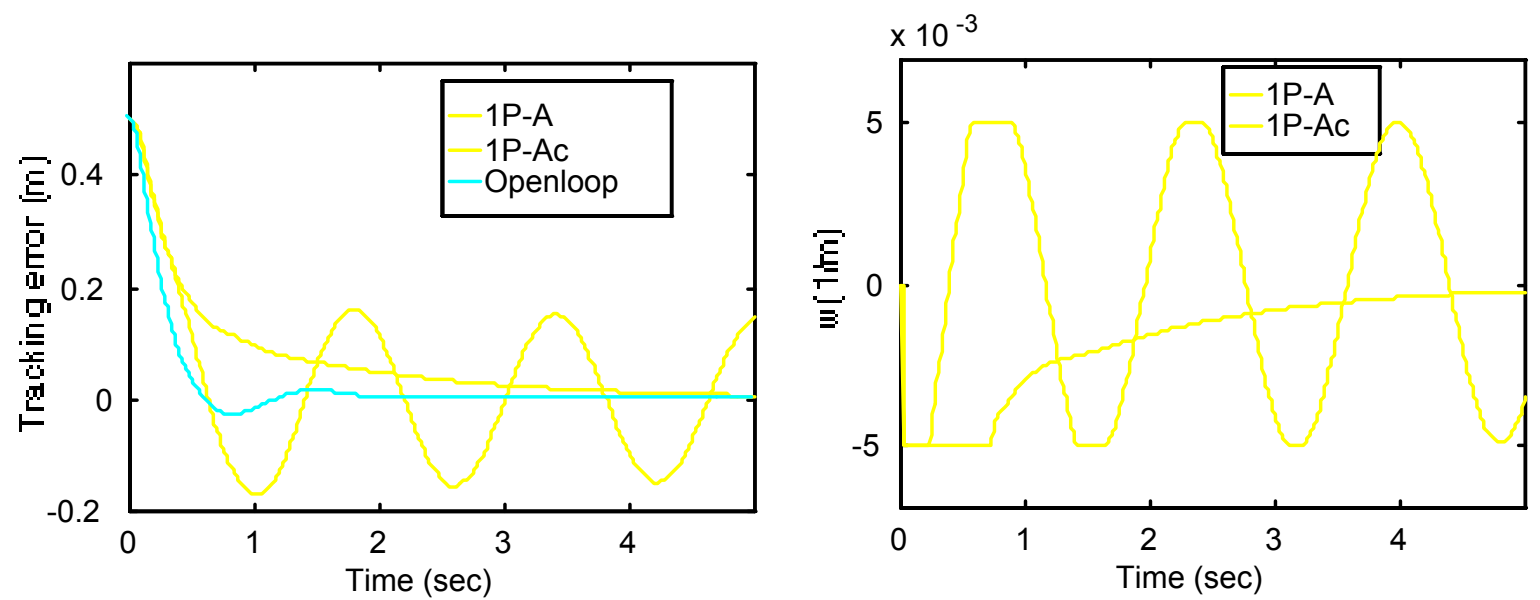

Figure 1 Simulation results of the $1 \mathrm{P}$ cases

Figure 2 shows the simulation results of the 1PP algorithm (disturbance advantaged case based on openloop A). The openloop (i.e. $\mathrm{W}=0$ ) as well as $1 \mathrm{P}$ (based on openloop A) cases are also presented for comparison. The disturbance law still does what intuition suggests. The disturbance signal switches even faster than the 1P-Ac case. This is an indication that the preview signal significantly improves the performance of the disturbance player. For this set of simulations, we have assumed that a time delay of $50 \mathrm{msec}$ exists between the steering angle command and the tire lateral force generation. Even with this short delay, the disturbance law did a better job than the 1P-Ac case (when the control law is exactly known to the disturbance player). It is important, however, to note action of the control player is delayed in the 1PP case. In other words, the plant dynamics of the $1 \mathrm{P}$ and $1 \mathrm{PP}$ cases are different.
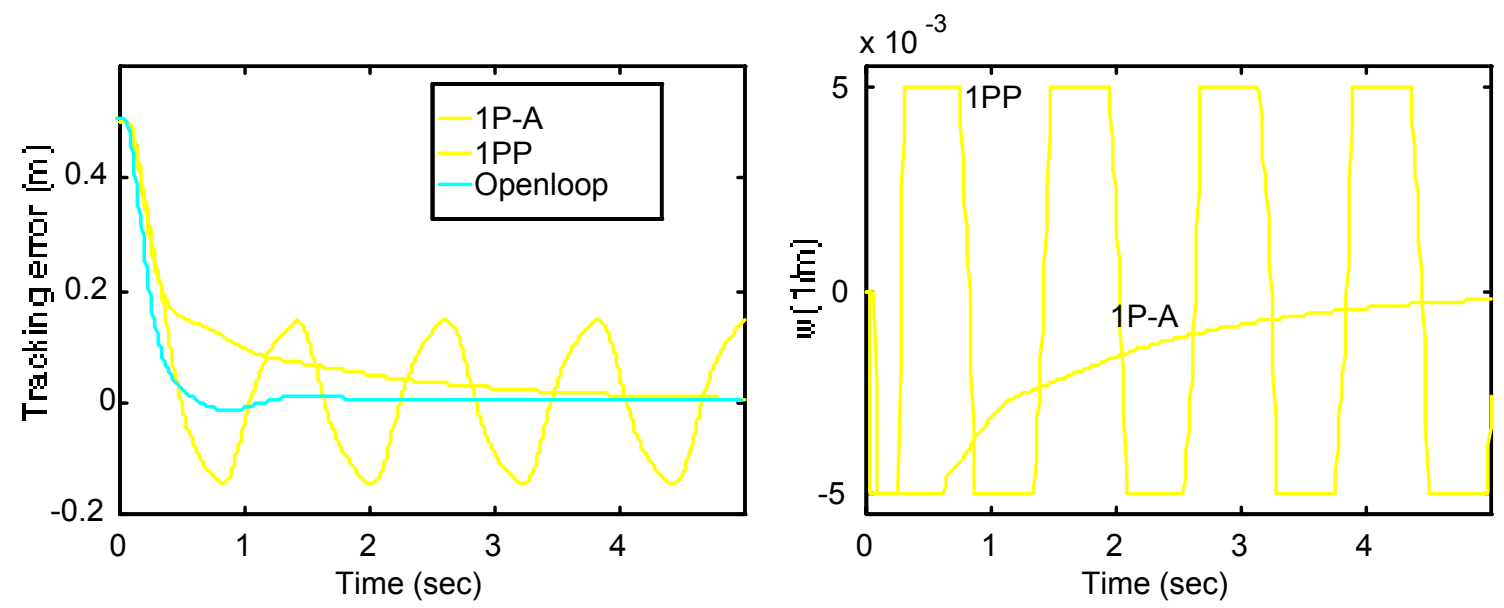

Figure 2 Simulation results of the 1PP case

Figure 3 shows the simulation results of the 2P algorithm. Two cases are presented. In the first case, both control and disturbance signals were generated from the $2 \mathrm{P}$ algorithm. In the second case, we substitute the $2 \mathrm{P}$ control algorithm by the LQ algorithm. It can be seen again that the disturbance signal switches between the two extreme values in the later case. The $2 \mathrm{P}$ control law stabilizes the vehicle quickly, while the LQ control law only achieves marginal stability. The point is that $2 \mathrm{P}$ algorithm can 
generate a disturbance player that is strong enough to defeat an ordinary control player (which, in this case, is based on LQ rather than $2 \mathrm{P}$ algorithm).
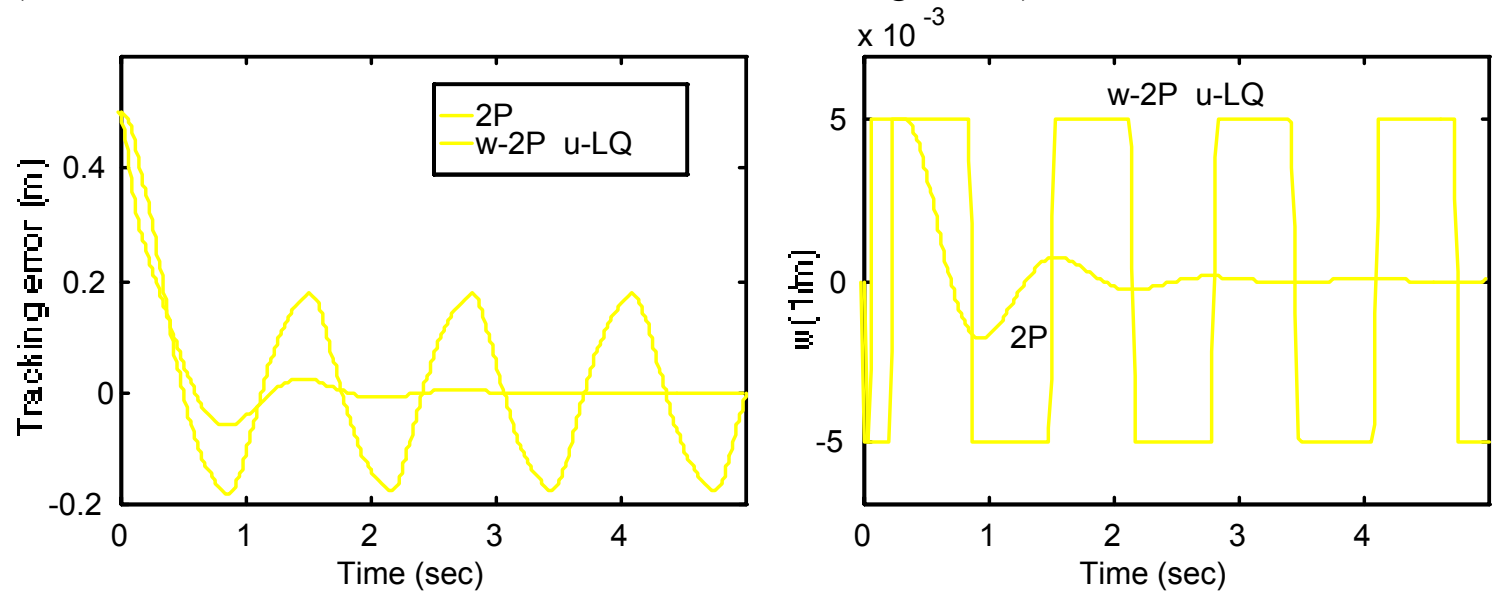

Figure 3 Simulation results of the $2 \mathrm{P}$ case

Figure 4 shows the $2 \mathrm{PP}$ case. The control law based on the $2 \mathrm{PP}$ algorithm is not very useful because the control law is so weak compared to the 2PP disturbance law that it simply gives up. The LQ control algorithm is used instead as the opponent for the disturbance laws. It can be seen that when preview information is available, the 2PP disturbance law performs even better than the $2 \mathrm{P}$ disturbance law. The preview time is again selected to be $50 \mathrm{msec}$. Even at such a short preview time, tracking error increases noticeably.
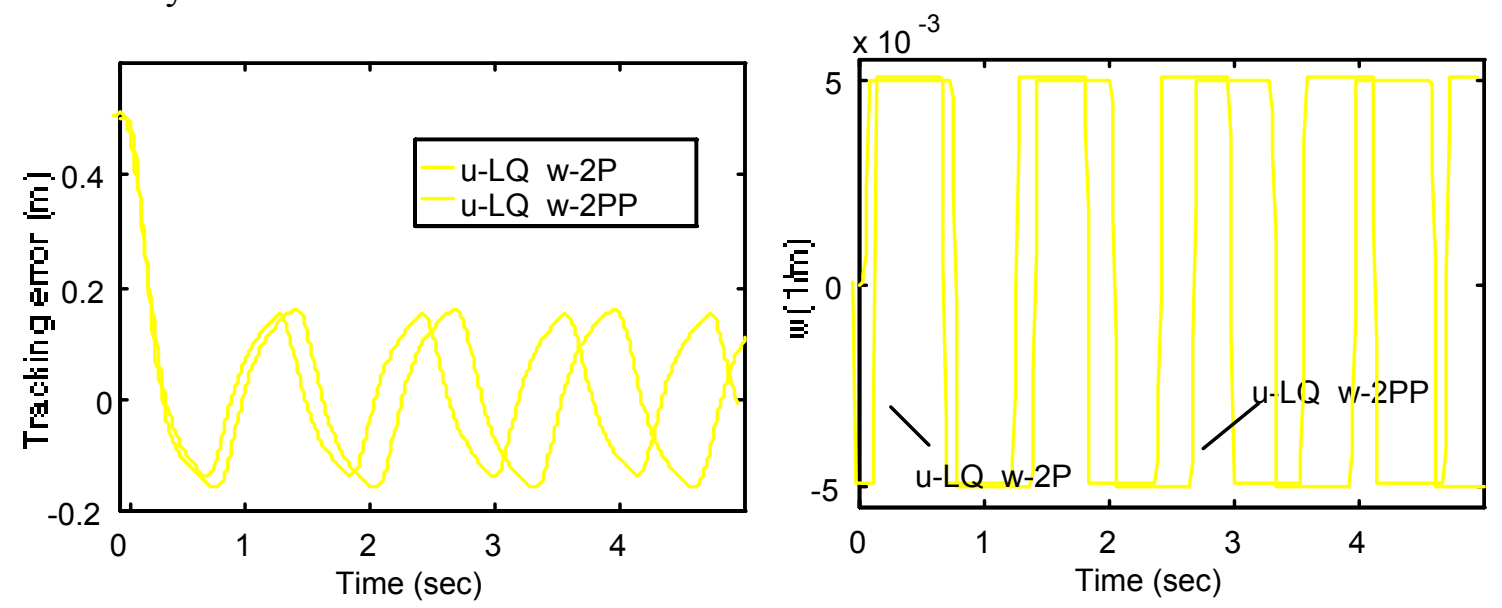

Figure 4 Simulation results of the $2 \mathrm{PP}$ case

Figures 5 and 6 show frequency-domain comparison of the four algorithms. We plug-in the disturbance laws into the dynamic equations, and obtain the closed-loop transfer functions from the control signal to output signals. Two output signals were selected, the lateral tracking error and the vehicle lateral acceleration. It can be seen that, compare to the openloop response $(\mathrm{w}=0)$, all the four proposed disturbance laws reduce the effectiveness of the control signal (reduced gain from $u$ to the tracking error $y_{s}$ ). More oscillation is generated and therefore the frequency response of the lateral acceleration transfer functions have higher magnitudes. The best performance is obviously achieved by the 2PP disturbance law. 

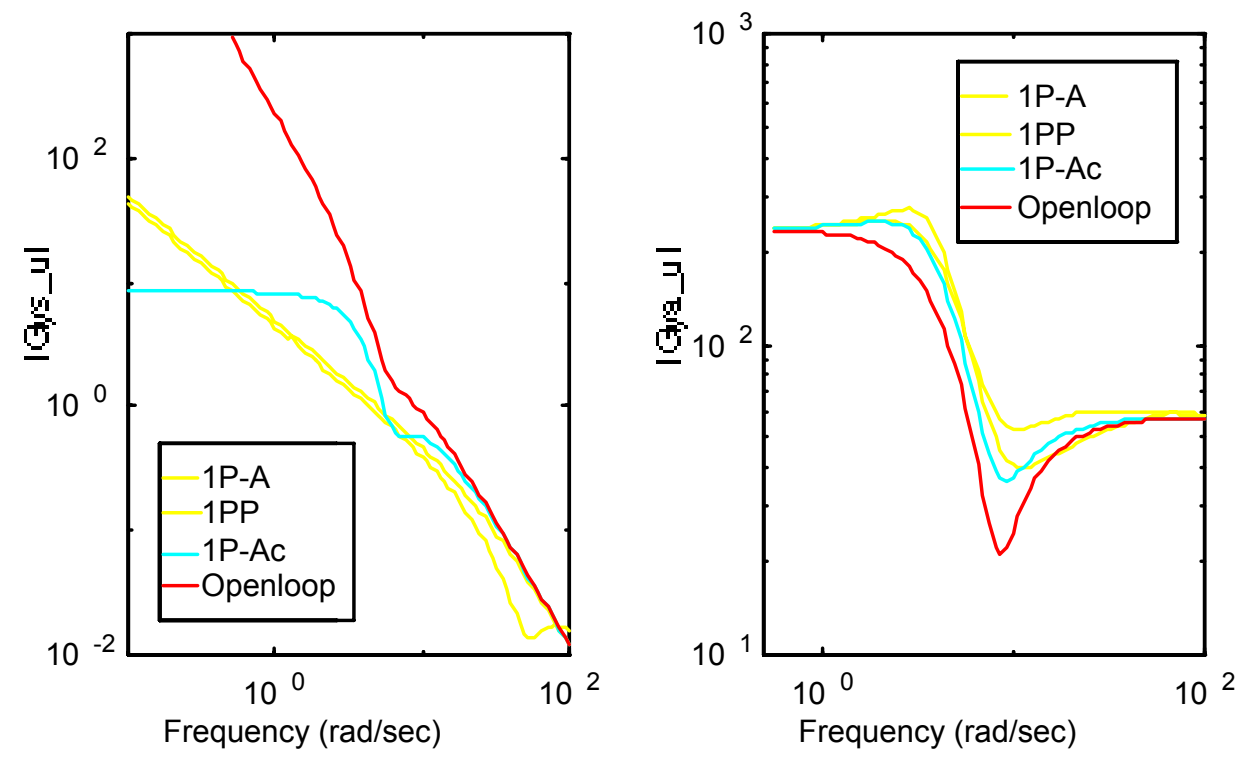

Figure 5 Frequency response of the $1 \mathrm{P}$ and $1 \mathrm{PP}$ cases
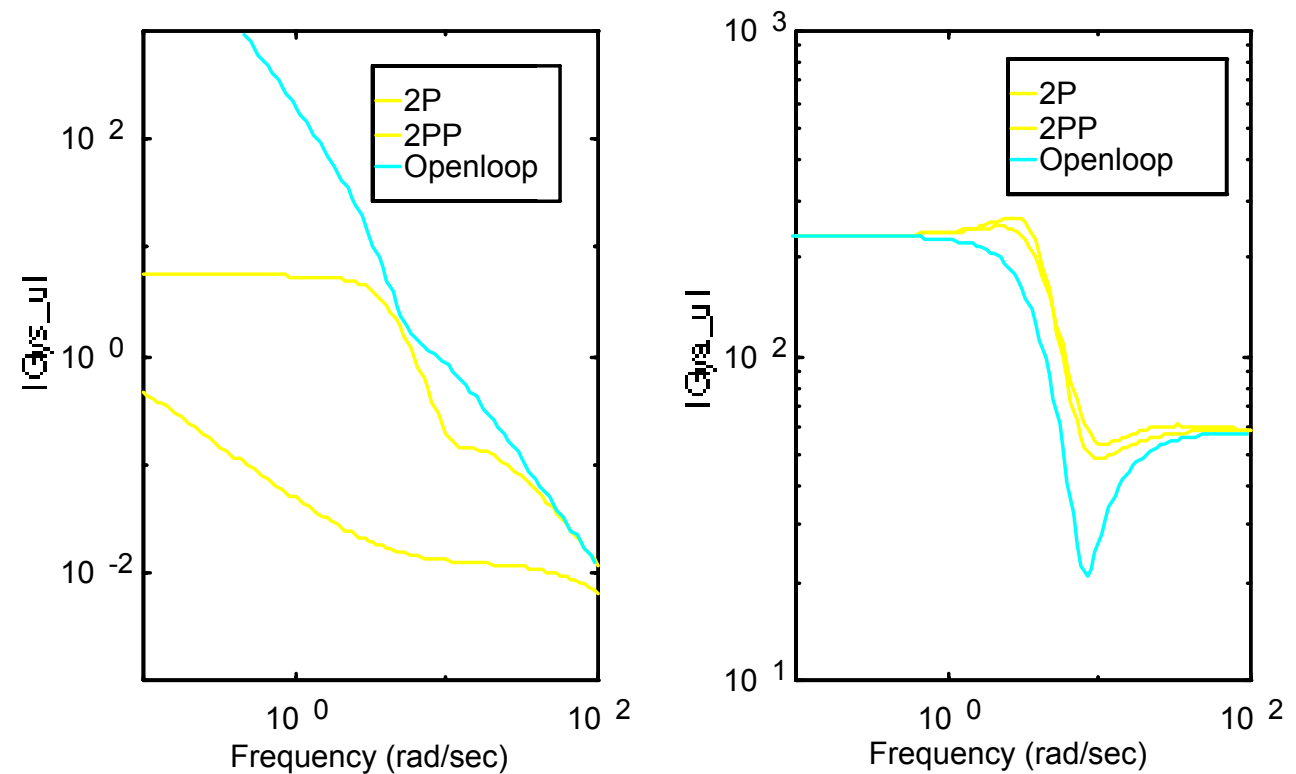

Figure 6 Frequency response of the 2P and 2PP cases

\subsection{Articulated truck rollover example}

The second application example deals with the rollover of an articulated vehicle. The plant is highly nonlinear, and include 79 state variables. All the simulations are obtained from ArcSim, a public-domain truck simulation software owned by the University of Michigan. This software simulates the Army M916A1/M870A2 vehicle, with detailed (and verified) nonlinear suspension, tire and steering models. Interested readers can download the ArcSim software and examine the rollover results from the University of Michigan web site http://arc.engin.umich.edu/sw_distri/arcsim.html.

The rollover problem is formulated as a $1 \mathrm{P}$ problem in this study. The steering wheel angle and the brake pressure are assumed to be controlled by the disturbance 
player, whose objective is to create rollover. Before showing the simulation results, we would like to emphasize the fact that if the excitation inputs (e.g. steering) are large enough, the truck will rollover, even under inputs of very simple forms. For example, it was found that at $60 \mathrm{mi} / \mathrm{hr}$, this truck rolls over under a step steering input of 150 degree, which translates to about 2.5 degree of steering at the front tires. In other words, when the disturbance input level is not limited, there are infinitely many maneuvers that are "bad". When the disturbance signal is limited to, say, 100 degrees. It is no longer a trivial task to come up with a steering/braking maneuver that rolls over the truck.

All the following simulation runs will assume an initial speed of $60 \mathrm{mi} / \mathrm{hr}$, unless otherwise stated. Due to the fact that the nonlinear vehicle model contains several hundred parameters, it is impossible to list all the parameter values. They can be found, however, from the downloadable ArcSim software. Figure 7 shows the vehicle response under two kinds of input excitations: a 130-degree step and a 130-degree sinusoidal steering. The frequency of the sinusoidal steering is selected to coincide with the natural frequency of the vehicle roll mode. The response of vehicle under the sinusoidal excitation is interesting, since the slalom test, frequently used to illustrate the rollover characteristic of passenger vehicles, are sinusoidal in nature. It is clear that the vehicle does not rollover under either maneuver. The maximum roll angle is only 7 degrees. The worst-case steering, identified by the nonlinear learning algorithm, however, easily rolls over the truck (see Figure 8).
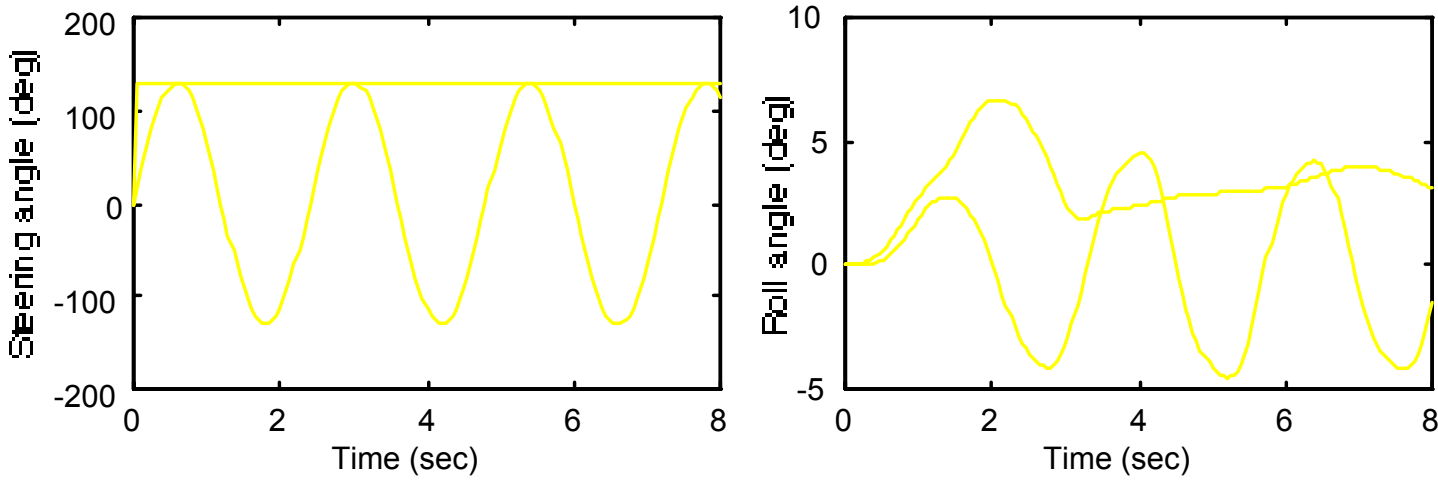

Figure 7 Vehicle response under 130 degree step and sinusoidal steering inputs, no brake
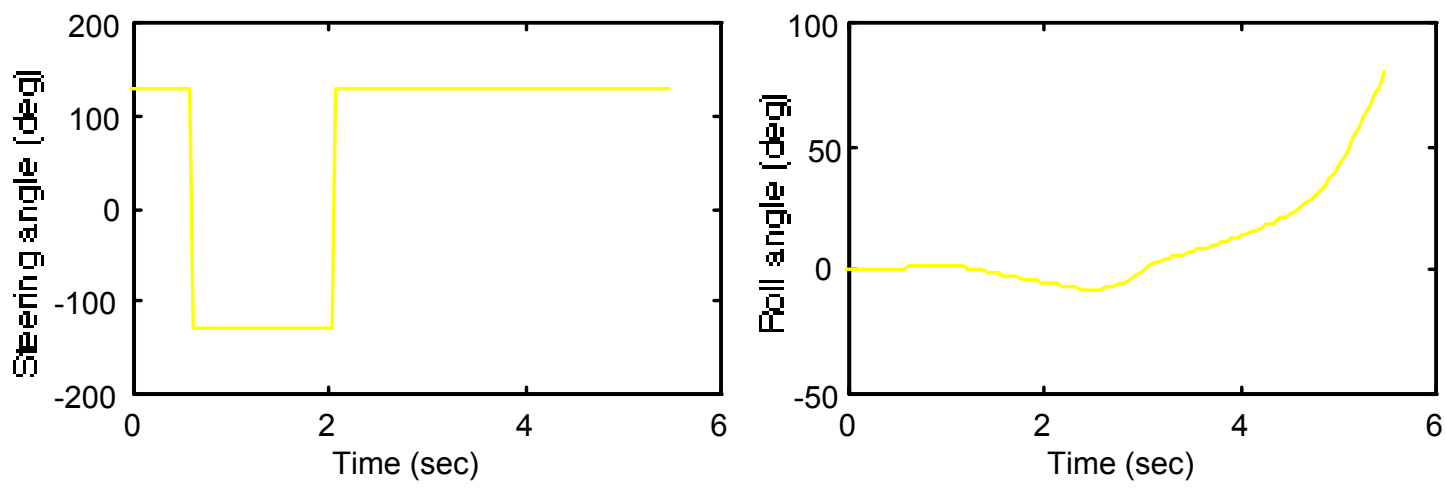

Figure 8 Vehicle response under 130 degree worst-case steering input, no brake 
When there are more than one input signals, no general engineering guideline guarantees inputs that work coherently with each other. Educated guesses even from experts are usually done in an ad hoc manner. One such coordination scheme has been proposed by UMTRI (The University of Michigan Transportation Research Institute) for the rollover of passenger vehicles (Dugoff et al. 1970). They proposed to steer the vehicle consistently to one side (a half-sinusoid), apply maximum brake when the vehicle roll rate becomes zero, and then switch the brake off when the roll rate crosses zero again. They applied this so-called "drastic maneuver" and successfully rolls over a passenger car (a Chevy Covair). In this paper, we borrow their idea and apply this "drastic maneuver" to this articulated truck.

When we limit the steering to 10 degrees, and brake to $120 \mathrm{psi}$, vehicle response of the corresponding "drastic maneuver" is shown in Figure 9. While the drastic maneuver is quite clever, most of the tricks are on manipulating the brake. The steering is pre-determined, and its potential has not been fully explored. The roll angle only reaches 3.8 degrees.
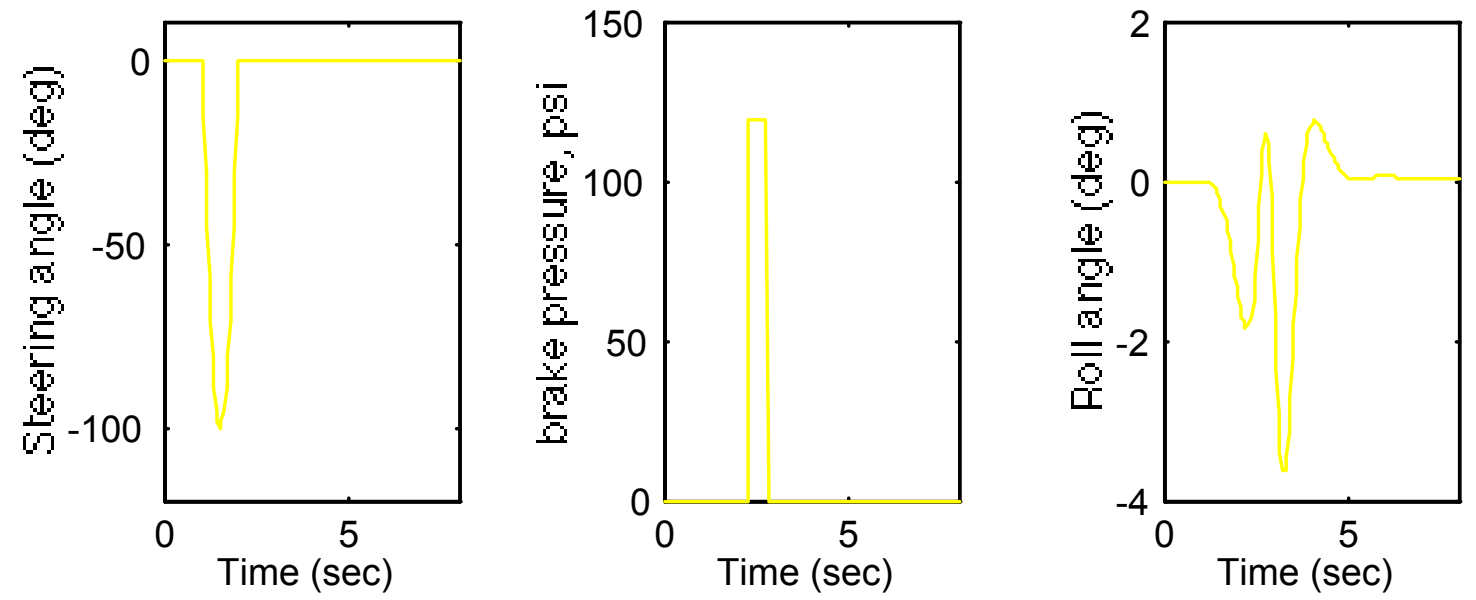

Figure 9 Vehicle response under the drastic maneuver

Under the same steering and brake limits, the vehicle response under the worstcase maneuver is shown in Figure 10. The input signals look very simple (switching functions) but the timing of the switching is non-trivial. The basic response of the vehicle is very similar to the UMTRI Drastic maneuver. However, the potential of both steering and brake are fully explored. The steering angle switches to help generating large roll angle. At about 4 seconds, the truck rolls over. The results can be best seen by examining Figure 10, as well as watching the animation results by actually running the ArcSim software. Figure 10 also include a shot from the ArcSim animator, which shows the truck rolling over by the worst-case maneuver. 

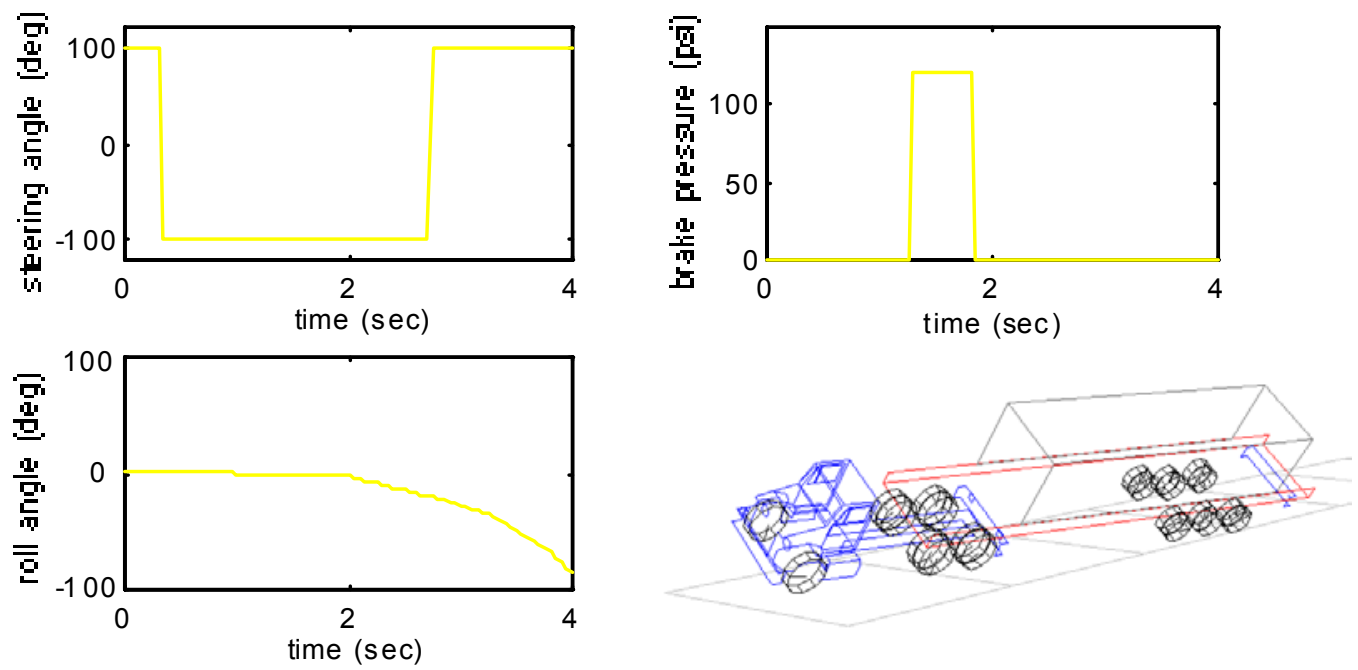

Figure 10 Vehicle response under the worst-case steering and braking maneuver

\section{Conclusions}

This paper presents an innovative worst-case evaluation methodology, which systematically generates worst-case disturbances for the evaluation of dynamic systems under extreme maneuvers. Four linear disturbance laws were presented: 1-player without preview (1P), 1-player with preview (1PP), 2-player without preview (2P), and 2-player with preview (2PP). These linear algorithms are exact dual, or simple extension of existing classical control and two-player game theories. When the dynamic system to be evaluated is nonlinear, these linear analytical results serve as initial guess for a nonlinear optimization algorithm. The final worst-case disturbance is then obtained numerically. An automatic vehicle steering control system was used to verify the effectiveness of the proposed algorithms. It was shown that when control player does not exist, or it exists and its behavior is well-known to the disturbance player, the disturbance signal generally switches between extreme values. The proposed methodology is applied to a complex behavior of the rollover of articulated trucks. It is shown that the most dangerous steering and braking excitation to rollover the truck is easily identified.

\section{Acknowledgment}

This research was supported by the U.S. Army TARDEC under the contract DAAE07-94-C-R094.

\section{References}

Basar, T. and Bernhard, P., $H_{\infty}$-Optimal Control and Related Minimax Design Problems--A Dynamic Game Approach, 2nd edition, Birkhauser, 1995.

Bryson, A. E., Ho, Y. C., Applied Optimal Control; Optimization, Estimation, and Control, Hemisphere Pub. Co., New York, 1975.

Dorato, P. and Drenick, R.F., "Optimality, Insensitivity, and Game Theory," in Sensitivity methods in control theory; proceedings of International Symposium on Sensitivity Analysis, 1964, Dubrovnik, Croatia. 
Dugoff, H., Ervin, R. D., Segel, L., "Vehicle Handling Test Procedures", Transportation Research Institute, University of Michigan, Report Contract No. FH-11-7297, November 1970.

Isidori, A. and Astofi, A., "Disturbance Attenuation and $H_{\infty}$ Control via Measurement Feedback in Nonlinear Systems," IEEE Trans. Auto. Contr., Vol.37, pp.1283-1293, 1992.

Lu, W.M. and Doyle, J.C., " $H_{\infty}$ Control of Nonlinear System via Output Feedback: A Class of Controllers," Proceeding of the 32nd Conference on Decision and Control, pp.166-171, 1993.

Ma, W. and Peng, H., "Worst-case Evaluation Methods for Vehicle Control Systems," Proceedings of the 1996 ASME International Congress and Exposition, Atlanta, GA, November 1996.

Peng, H. and Tomizuka, M., "Preview Control for Vehicle Lateral Guidance in Highway Automation," ASME J. of Dynamic Systems, Measurement and Control, Vol.115, No.4, Dec., 1993, pp.679-686.

Tomizuka, M., Whitney, D.E., "Optimal Discrete Finite Preview Problems (Why and How is Future Information Important?)" Transactions of ASME Journal of Dynamic Systems, Measurement and Control, Dec. 1975, pp.319-325.

Tomizuka, M., "Optimal Continuous Finite Preview Problem," IEEE Transactions on Automatic Control, Vol.AC-20, No.3, pp.362-365, June. 1975.

Tomizuka, M., "The Continuous Optimal Finite Preview Control Problem," Transactions of the Society of Instrument and Control Engineers, Vol.12, No.1, pp.7-12, Feb. 1976.

Van der Schaft, A. J., " $L_{2}$-Gain Analysis of Nonlinear Systems and Nonlinear State Feedback $H_{\infty}$ Control," IEEE Trans. Auto, Contr., Vol.37, No.6, pp.770-784, June 1992.

Von Glasner, E-C., "Active Safety of Commercial Vehicles," Proceedings of the International Symposium on Advanced Vehicle Control (AVEC) 1994, pp.9-14.

Witsenhausen, H.S., "A Minimax Control Problem for Sampled Linear Systems," IEEE Transactions for Automatic Control, AC-13:5-21, February 1968.

Yaesh, I. and Shaked, U., "Minimum $H_{\infty}$-norm Regulation of Linear Discrete-time Systems and its Relation to Linear Quadratic Difference Games," Proceedings of the 29th IEEE Conference of Decision and Control, 1989, pp.942-947. 